\title{
SOLUTION OF THE CONGRUENCE PROBLEM FOR ARBITRARY HERMITIAN AND SKEW-HERMITIAN MATRICES OVER POLYNOMIAL RINGS
}

\author{
Dragomir Ž. Đoković And FERnANDo Szechtman
}

\begin{abstract}
Let ${ }^{*}$ be the involutorial automorphism of the complex polynomial algebra $\mathbf{C}[t]$ which sends $t$ to $-t$. Answering a question raised by V.G. Kac, we show that every hermitian or skew-hermitian matrix over this algebra is congruent to the direct sum of $1 \times 1$ matrices and $2 \times 2$ matrices with zero diagonal. Moreover we show that if two $n \times n$ hermitian or skew-hermitian matrices have the same invariant factors, then they are congruent. The complex field can be replaced by any algebraically closed field of characteristic $\neq 2$.
\end{abstract}

\section{Introduction}

Let $R$ be the polynomial algebra $F[t]$ in one variable $t$ over an algebraically closed field $F$ of characteristic $\neq 2$. Let ${ }^{*}$ denote the involution of $R$ which is the identity on $F$ and sends $t$ to $-t$. (We remark that all nontrivial $F$-involutions of $F[t]$ are conjugate in $\operatorname{Aut}_{F}(F[t])$.) It induces the $Z_{2}$-gradation $R=R_{0} \oplus R_{1}$ of $R$ with $R_{0}=F\left[t^{2}\right]$ and $R_{1}=t R_{0}$. We shall refer to the elements of $R_{0}$ (resp. $\left.R_{1}\right)$ as even (resp. odd).

Let $M_{n}(R)$ denote the algebra of $n$ by $n$ matrices over $R$. If $A=\left(a_{i j}\right) \in$ $M_{n}(R)$, we define $A^{*}$ to be the matrix $B=\left(b_{i j}\right) \in M_{n}(R)$ where $b_{i j}=a_{j i}^{*}$. Thus ${ }^{*}$ is now made into an involution of $M_{n}(R)$. We say that $A \in M_{n}(R)$ is hermitian (resp. skew-hermitian) if $A^{*}=A$ (resp. $A^{*}=-A$ ). Two hermitian (resp. skew-hermitian) matrices $A, B \in M_{n}(R)$ are said to be congruent if $B=S^{*} A S$ for some $S \in \mathrm{GL}_{n}(R)$.

Not long ago V.G. Kac [3] posed the following question to the first author (see also [1]).

If $F$ is the complex field, is it true that every hermitian or skew-hermitian matrix $A \in M_{n}(R)$ is congruent to the direct sum of $1 \times 1$ matrices and $2 \times 2$ matrices with zero diagonal?

Note that no condition is imposed on the determinant of $A$. (The usual restriction is that $A$ be unimodular.) The two cases, hermitian and skew-hermitian, of this problem are tightly linked because if $A$ is hermitian then $t A$ is skewhermitian, and vice versa.

Received June 11, 2002.

2000 Mathematics Subject Classification. Primary 15A57; Secondary 15A63. 
The main objective of our paper is to give an affirmative answer to Kac's question (Theorem 4.3), which we find quite surprising. The case $n=2$ is dealt with in Section 3 and the general case in Section 4. In Section 4, we also prove that two hermitian (or skew-hermitian) matrices $A, B \in M_{n}(R)$ are congruent if and only if they have the same invariant factors (Theorem 4.5). Then, in Section 5 , we are able to characterize the sequence of invariant factors of a hermitian or skew-hermitian matrix, and to give the canonical form under congruence for such a matrix. In the last section we make comments on other fields and characterize those for which Kac's question has positive answer.

The authors would like to thank Prof. Kac for his interest in our work and for proposing this interesting problem. We also thank Prof. L. Vaserstein for his comments on an earlier version of this paper.

\section{Preliminaries}

The elements $a \in R$ are polynomials and so they can be evaluated at any point $\lambda \in F$. We denote by $a(\lambda)$ the value of $a$ at $\lambda$. We say that a nonzero element $a \in R$ is pure if $\operatorname{gcd}\left(a, a^{*}\right)=1$. If $a, b \in R$ with $a$ pure and $b$ even (resp. odd), then there exists $x \in R$ such that $a x+a^{*} x^{*}=b$ (resp. $a x-a^{*} x^{*}=b$ ). (Choose $y, z \in R$ such that $a y+a^{*} z=b / 2$ and set $x=y+z^{*}\left(\right.$ resp. $\left.x=y-z^{*}\right)$.) Since $F$ is algebraically closed, each $a \in R_{0}$ can be written as $a=b b^{*}, b \in R$.

If $I=R a$ is a homogeneous (i.e., *-invariant) ideal of $R$, then its generator $a$ is also homogeneous, i.e., it is either even or odd. If $A=\left(a_{i j}\right) \in M_{n}(R)$ is hermitian or skew-hermitian, then the ideal generated by all entries $a_{i j}$ is *-invariant and we denote its generator by $\operatorname{gcd}(A)$. Hence $\operatorname{gcd}(A)$ is the first invariant factor of $A$.

Let us fix a hermitian or skew-hermitian matrix $A \in M_{n}(R)$. Let $R^{n}$ denote the free $R$-module of rank $n$ consisting of column vectors. We shall denote by $e_{1}, \ldots, e_{n}$ the standard basis vectors of $R^{n}$. The matrix $A$ defines a hermitian or skew-hermitian form $f_{A}: R^{n} \times R^{n} \rightarrow R$ by

$$
f_{A}(v, w)=v^{*} A w .
$$

By [2, Lemma 1], $A$ is congruent to the direct sum of a zero matrix and a hermitian or skew-hermitian matrix with nonzero determinant. (The proof given there in the hermitian case is also valid in the skew-hermitian case.) This argument shows that it suffices to consider only the hermitian or skew-hermitian matrices with nonzero determinant.

As $F$ is algebraically closed, if $n \geq 2$ there exist nonzero isotropic vectors, i.e., nonzero vectors $v \in R^{n}$ such that $f_{A}(v, v)=0$. At the referee's suggestion, we include a proof. Clearly, it suffices to consider the hermitian case (otherwise replace $A$ by $t A$ ). We may also assume that $n=2$. Then let

$$
A=\left(\begin{array}{cc}
a & b \\
b^{*} & c
\end{array}\right)
$$


and we may assume that $a \neq 0$. As $b b^{*}-a c$ is even, it can be written as $d d^{*}$ for some $d \in R$. Then $(d-b) e_{1}+a e_{2}$ is a nonzero isotropic vector.

Assume that $\operatorname{det}(A) \neq 0$ and set $d=\operatorname{gcd}(A)$. Then $A=d B$ for some matrix $B \in M_{n}(R)$ such that $B^{*}= \pm B$ and $\operatorname{gcd}(B)=1$. Therefore, without any loss of generality, we may assume that $\operatorname{det}(A) \neq 0$ and $\operatorname{gcd}(A)=1$.

\section{The case $n=2$}

In this section we show that the answer to Kac's question is affirmative if $n=2$. We start with the hermitian case.

Proposition 3.1. If $A=A^{*} \in M_{2}(R)$, $\operatorname{det}(A) \neq 0$, and $\operatorname{gcd}(A)=1$, then $A$ is congruent to $\operatorname{diag}(1, \operatorname{det}(A))$.

Proof. Since there exist nonzero isotropic vectors, we may assume that

$$
A=\left(\begin{array}{cc}
0 & a \\
a^{*} & b
\end{array}\right) \text {. }
$$

The element $a_{0}=\operatorname{gcd}\left(a, a^{*}\right)$ is homogeneous, i.e., $a_{0}^{*}= \pm a_{0}$. We have a factorization $a=a_{0} a_{1}$ where $a_{1}$ is pure. By the hypothesis, $\operatorname{gcd}\left(a_{0}, b\right)=1$. Consequently, there exist homogeneous elements $x$ and $y$, with $y$ even, such that $a_{0} x+b y=1$. Clearly we may assume that $y(0) \neq 0$. Choose a factorization $y=z z^{*}$ such that $a_{1} z$ is pure. Then there exists $w \in R$ such that

$$
a_{1} z w+a_{1}^{*} z^{*} w^{*}=1 .
$$

Since $a_{0} x$ is even, we find that

$$
\begin{aligned}
1 & =a_{0} x+b y \\
& =a_{0} x\left(a_{1} z w+a_{1}^{*} z^{*} w^{*}\right)+b z z^{*} \\
& =a x w z+a^{*} x^{*} w^{*} z^{*}+b z z^{*} \\
& =f_{A}\left(x^{*} w^{*} e_{1}+z e_{2}, x^{*} w^{*} e_{1}+z e_{2}\right) .
\end{aligned}
$$

The assertion of the proposition is now obvious.

Next we consider the skew-hermitian case. We shall need the following simple lemma.

Lemma 3.2. Let $a, b \in R$ satisfy $\operatorname{gcd}(a, b)=\operatorname{gcd}\left(b, b^{*}\right)=1$. Then there exist $x, y \in R$, with $x$ even, such that $a x+b y=1$.

Proof. Choose $u, v \in R$ such that $a u+b v=1$ and $z \in R$ such that $b z-b^{*} z^{*}=$ $u^{*}-u$. Then $x=u+b z \in R_{0}$ and $y=v-a z$ satisfy $a x+b y=1$.

Proposition 3.3. Let $A \in M_{2}(R), A^{*}=-A$, $\operatorname{det}(A) \neq 0$, and $\operatorname{gcd}(A)=1$. Then $A$ is congruent to a matrix with zero diagonal. 
Proof. We may assume that

$$
A=\left(\begin{array}{cc}
0 & a \\
-a^{*} & b
\end{array}\right)
$$

As $a(0) \neq 0$, we can write $a=a_{1} c c^{*}$ with $a_{1} c$ pure. By Lemma 3.2, there exist $x, d \in R$, with $x$ even, such that

$$
b x+c d=1 .
$$

By replacing $(x, d)$ with $\left(x+\lambda c c^{*}, d-\lambda b c^{*}\right)$, where $\lambda \in F$ is suitably chosen, we may assume that $\operatorname{gcd}\left(a_{1}, x\right)=1$. Since $b$ is odd, we can choose $w \in R$ such that $c^{*} w^{*}-c w=b$. Since $\operatorname{gcd}\left(a_{1}^{*}, c x\right)=1$, there exist $v, p \in R$ such that

$$
a_{1}^{*} v-c x p^{*}=x w^{*}-d^{*} .
$$

Choose $q \in R$ such that

and set

$$
a_{1}^{*} q-a_{1} q^{*}=p-p^{*}
$$

$$
y=v+c x q, \quad z=w+c^{*}\left(p+a_{1} q^{*}\right) .
$$

Then

$$
\begin{aligned}
c^{*} z^{*}-c z & =c^{*} w^{*}+c c^{*}\left(p^{*}+a_{1}^{*} q\right)-c w-c c^{*}\left(p+a_{1} q^{*}\right) \\
& =c^{*} w^{*}-c w=b \\
x z^{*}-a_{1}^{*} y & =x w^{*}+c x\left(p^{*}+a_{1}^{*} q\right)-a_{1}^{*} v-a_{1}^{*} c x q \\
& =c x p^{*}+x w^{*}-a_{1}^{*} v=d^{*} .
\end{aligned}
$$

Hence

$$
\begin{aligned}
a_{1}^{*} c^{*} y & =c^{*} x z^{*}-c^{*} d^{*}=c^{*} x z^{*}-(1+b x) \\
& =x\left(c^{*} z^{*}-b\right)-1=c x z-1
\end{aligned}
$$

and so

We have

$$
S=\left(\begin{array}{ll}
c x & y \\
a_{1}^{*} c^{*} & z
\end{array}\right) \in \mathrm{SL}_{2}(R) .
$$

where

$$
S^{*}\left(\begin{array}{cc}
0 & a_{1} c^{2} \\
-a_{1}^{*}\left(c^{*}\right)^{2} & 0
\end{array}\right) S=\left(\begin{array}{cc}
0 & r \\
-r^{*} & s
\end{array}\right),
$$

$$
\begin{aligned}
r & =a_{1} c c^{*}\left(c x z-a_{1}^{*} c^{*} y\right)=a_{1} c c^{*}=a, \\
s & =a_{1} c^{2} y^{*} z-a_{1}^{*}\left(c^{*}\right)^{2} y z^{*} \\
& =a_{1} c y^{*} \cdot c z-a_{1}^{*} c^{*} y \cdot c^{*} z^{*} \\
& =\left(c^{*} x z^{*}-1\right) c z-(c x z-1) c^{*} z^{*} \\
& =c^{*} z^{*}-c z=b .
\end{aligned}
$$

Hence

$$
\left(\begin{array}{cc}
0 & r \\
-r^{*} & s
\end{array}\right)=A \text {. }
$$




\section{Equivalence implies congruence}

The first main theorem is a simple consequence of the following two propositions. The first one deals with hermitian matrices.

Proposition 4.1. If $A=\left(a_{i j}\right) \in M_{n}(R), A^{*}=A$ and $\operatorname{gcd}(A)=1$, then there exists $v \in R^{n}$ such that $f_{A}(v, v)=1$.

Proof. We may assume that $\operatorname{det}(A) \neq 0$. The proof will be by induction on $n$. The case $n=1$ is obvious. For the case $n=2$ see Proposition 3.1. Thus let $n>2$.

Since there exist nonzero isotropic vectors, we may assume that $a_{11}=0$. Moreover, we may assume that $a_{1 j}=0$ for $j<n$. Denote by $E_{i j}$ the matrix of order $n$ whose $(i, j)$-th entry is 1 and all other entries are 0 , and by $I_{n}$ the identity matrix. For any $\lambda \in F$, the matrix

$$
A_{\lambda}=\left(I_{n}+\lambda E_{21}\right) A\left(I_{n}+\lambda E_{12}\right)
$$

is congruent to $A$. Let $A_{\lambda}^{\prime}$ denote the submatrix of $A_{\lambda}$ obtained by deleting the first row and column. Set $A^{\prime}=A_{0}^{\prime}$. Note that for $\lambda, \mu \in F$ we have

$$
A_{\lambda}^{\prime}-A_{\mu}^{\prime}=(\lambda-\mu)\left(a_{1 n} E_{1, n-1}+a_{n 1} E_{n-1,1}\right),
$$

where now $E_{i j}$ 's have order $n-1$.

As $\operatorname{det}(A) \neq 0$, we have $a_{r s} \neq 0$ for some $r, s \in\{2,3, \ldots, n-1\}$. Since $a_{r s}$ has only finitely many monic divisors, there exist $\lambda, \mu \in F$, with $\lambda \neq \mu$, such that $\operatorname{gcd}\left(A_{\lambda}^{\prime}\right)=\operatorname{gcd}\left(A_{\mu}^{\prime}\right)$. Denote this common $\operatorname{gcd}$ by $d$. The displayed formula for $A_{\lambda}^{\prime}-A_{\mu}^{\prime}$ shows that $d$ divides $a_{1 n}$ (and $a_{n 1}$ ). It also divides all entries of $A^{\prime}$. Since $a_{1 j}=0$ for $j<n$, it follows that $d$ divides all entries of $A$. As $\operatorname{gcd}(A)=1$, we conclude that $d=1$.

We have shown that $\operatorname{gcd}\left(A_{\lambda}^{\prime}\right)=1$ for some $\lambda \in F$. By the induction hypothesis there exists $w \in R^{n-1}$ such that $f_{A_{\lambda}^{\prime}}(w, w)=1$. As $A$ and $A_{\lambda}$ are congruent, there exists $v \in R^{n}$ such that $f_{A}(v, v)=1$.

The second proposition is a skew-hermitian analog of the first one. We shall need the following definition. Let $\nu_{A}$ denote the minimum degree of nonzero polynomials $f_{A}(v, w)$ over all $v, w \in R^{n}$ with $f_{A}(v, v)=0$.

Proposition 4.2. If $A=\left(a_{i j}\right) \in M_{n}(R), A^{*}=-A$ and $\operatorname{gcd}(A)=1$, then $A$ is congruent to the direct sum $B \oplus D$, where

$$
B=\left(\begin{array}{cc}
0 & f \\
-f^{*} & 0
\end{array}\right),
$$

with $f$ pure of degree $\nu_{A}$. Furthermore, $f f^{*}$ divides all entries of $D$, i.e., $\operatorname{det}(B)$ is the second invariant factor of $A$.

Proof. For the case $n=2$ see Proposition 3.3. Thus let $n>2$.

After a suitable change of basis, we may assume that $f_{A}\left(e_{1}, e_{1}\right)=0$ and that there exists $w \in R^{n}$ such that $f_{A}\left(e_{1}, w\right)$ is nonzero and has degree $\nu_{A}$. Thus 
$a_{11}=0$. By performing some additional elementary congruence transformations, we may also assume that $a_{12} \neq 0$ has degree $\nu_{A}$ and that $a_{1 j}=0$ for $j>2$.

Denote by $\mathcal{A}$ the set of all skew-hermitian matrices $X=\left(x_{i j}\right) \in M_{n}(R)$ which are congruent to $A$ and such that $x_{1 j}=0$ for $j \neq 2$ while $x_{12}$ has degree $\nu_{A}$. For $X \in \mathcal{A}$ let $d_{X}=\operatorname{gcd}\left(x_{12}, x_{21}, x_{22}\right)$ where we require $d_{X}$ to be monic. Let $\mathcal{A}_{0}$ denote the set of all $X \in \mathcal{A}$ such that $d_{X}$ has the minimum degree. Without any loss of generality, we assume that $A \in \mathcal{A}_{0}$.

Our first objective is to show that $d_{A}$ is 1 or $t$. Let $2 \leq r<s \leq n$ and for $x \in R$ define $A_{x} \in \mathcal{A}$ by

$$
A_{x}=\left(I_{n}+x^{*} E_{r s}\right) A\left(I_{n}+x E_{s r}\right)
$$

and set $d_{x}=d_{A_{x}}$. For $\lambda \in F$, the $(r, r)$-th entry of $A_{\lambda x}$ is

$$
a_{r r}+\lambda\left(a_{r s} x^{*}-a_{r s}^{*} x\right)+\lambda^{2} a_{s s} x x^{*} .
$$

We take first $r=2$. As $a_{12}$ has only finitely many monic divisors, we can choose distinct $\alpha, \beta, \gamma \in F$ such that $d_{\alpha x}=d_{\beta x}=d_{\gamma x}$. Denote this common gcd by $d$. As the Vandermonde determinant of $\alpha, \beta, \gamma$ is not $0, d$ must divide $a_{22}$, $a_{2 s} x^{*}-a_{2 s}^{*} x$ and $a_{s s} x x^{*}$. It follows that $d$ divides $d_{A}$, and consequently we must have $d=d_{A}$. By taking $x=1$, we infer that $d_{A}$ divides the diagonal entries of $A$. As $d_{A}$ divides $a_{2 s} x^{*}-a_{2 s}^{*} x$ for all $x \in R$, we deduce that $d_{A}$ divides $t a_{2 s}$.

Next we take $r>2$. Since $d_{A}$ must divide (4.2) for all $\lambda \in F$ and $x \in R$, we infer that $d_{A}$ divides $a_{r s} x^{*}-a_{r s}^{*} x$ for all $x \in R$. Consequently, $d_{A}$ divides $t a_{r s}$. As $\operatorname{gcd}(A)=1$, it follows that $d_{A}$ is either 1 or $t$.

We shall now rule out the possibility $d_{A}=t$. Suppose that $d_{A}=t$. Assume that all entries $a_{2 j}$ are divisible by $t$. In the above construction, we take once again $r=2$ and choose $s>2$ such that $a_{s k}$ is not divisible by $t$ for some $k>1$ and $k \neq s$. As above, we can choose a nonzero $\lambda \in F$ such that $d_{\lambda}=d_{A}$. Then the $(2, k)$-th entry of $A_{\lambda}$ is not divisible by $t$. Hence we can assume that one of the entries in the second row, say $a_{23}$, is not divisible by $t$.

The $3 \times 3$ submatrix in the upper left hand corner of $A$ has the form

$$
\left(\begin{array}{ccc}
0 & a t^{k} & 0 \\
-a^{*}(-t)^{k} & b t & c \\
0 & -c^{*} & d
\end{array}\right),
$$

where $a, b, c$ are not divisible by $t, k \geq 1, \operatorname{gcd}\left(a, a^{*}, b\right)=1$, and the degree of $a t^{k}$ is equal to $\nu_{A}$. Moreover, by using Proposition 3.1, we may also assume that $a$ is pure. Hence we can choose $x \in R$ such that $a x^{*}+a^{*} x=(-1)^{k} b$. Then the vector $v=x e_{1}+t^{k-1} e_{2}$ is isotropic and

$$
f_{A}\left(v, e_{1}\right)=a^{*} t^{2 k-1}, \quad f_{A}\left(v, e_{3}\right)=c(-t)^{k-1} .
$$

Hence there exists $w \in R^{n}$ such that

$$
f_{A}(v, w)=t^{k-1} \operatorname{gcd}\left(a^{*}, c\right),
$$

contradicting the fact that $a t^{k}$ has degree $\nu_{A}$. We conclude that $d_{A}=1$. 
It is now easy to finish the proof. By Proposition 3.3, we may assume that the $2 \times 2$ submatrix $B$ in the upper left hand corner of $A$ has the form (4.1) with $f$ pure. From the definition of $\nu_{A}$ it follows that the entries $a_{1 j}, j>2$, are divisible by $f$. By performing suitable elementary congruence transformations, we may assume that all these entries are 0. A similar argument can be used to make the entries $a_{2 j}=0$ for $j>2$.

Thus we have $A=B \oplus D$ where $D=\left(d_{i j}\right)$. Replace the zero in the $(2,2)$ position of $A$ by $-d_{i i}$. It follows from Proposition 3.3 that this change can be achieved by a congruence transformation on the block $B$. Now add the $(i+2)$-nd row of $A$ to the second row and then the $(i+2)$-nd column to the second column. The entry in the $(2,2)$ position will become 0 again. From the definition of $\nu_{A}$ it follows that the $(2, j+2)$-nd entry of this new matrix must be divisible by $f^{*}$. As this entry is equal to $d_{i j}$, we conclude that all entries of $D$ are divisible by $f^{*}$. As $D^{*}=-D$, they are also divisible by $f$. As $f$ is pure, all entries of $D$ are divisible by $f f^{*}$.

We are now able to answer Kac's question.

Theorem 4.3. If $A \in M_{n}(R)$ is hermitian or skew-hermitian, then $A$ is congruent to the direct sum of $1 \times 1$ matrices and $2 \times 2$ matrices with zero diagonal.

Proof. As observed in Section 2, we may assume that $\operatorname{det}(A) \neq 0$ and $\operatorname{gcd}(A)=$ 1 . We already know that the theorem is true if $n \leq 2$. It remains to use induction and apply the Propositions 4.1 and 4.2.

To prove our second main result, we need the following simple lemma.

Lemma 4.4. Let $A, B \in M_{2}(R)$ be skew-hermitian, $\operatorname{gcd}(A)=\operatorname{gcd}(B)=1$, and $\operatorname{det}(A)=\operatorname{det}(B) \neq 0$. Then $A$ and $B$ are congruent.

Proof. By Proposition 3.3, we may assume that

$$
A=\left(\begin{array}{cc}
0 & a b \\
-a^{*} b^{*} & 0
\end{array}\right), \quad B=\left(\begin{array}{cc}
0 & a b^{*} \\
-a^{*} b & 0
\end{array}\right),
$$

with $a b$ and $a b^{*}$ pure. There exist $x, y \in R_{0}$ such that $b b^{*} x-a a^{*} y=1$. If

$$
S=\left(\begin{array}{cc}
b^{*} x & a y \\
a^{*} & b
\end{array}\right)
$$

then $S \in \mathrm{SL}_{2}(R)$ and $S^{*} B S=A$.

Recall that two matrices $A, A^{\prime} \in M_{n}(R)$ are said to be equivalent if there exist $S, T \in \mathrm{GL}_{n}(R)$ such that $A^{\prime}=S A T$. A necessary and sufficient condition for $A$ and $A^{\prime}$ to be equivalent is that they have the same invariant factors.

Theorem 4.5. Let $A, A^{\prime} \in M_{n}(R)$ be both hermitian or both skew-hermitian. If $A$ and $A^{\prime}$ are equivalent, then they are congruent. 
Proof. We use induction on $n$. The case $n=1$ is trivial. Let $n>1$. Denote the invariant factors of $A$ (and $A^{\prime}$ ) by $f_{1}, \ldots, f_{n}$. If $f_{n}=0$ then we can use the induction hypothesis. Assume that $f_{n} \neq 0$. By dividing $A$ and $A^{\prime}$ by $f_{1}$, we may assume that $f_{1}=1$.

Now if $A$ and $A^{\prime}$ are hermitian (resp. skew-hermitian) then Proposition 4.1 (resp. Proposition 4.2 and Lemma 4.4) allows us to finish the proof by using the induction hypothesis.

We shall give more details in the skew-hermitian case. By Proposition 4.2 we may assume that $A=B \oplus D$, where $B$ and $D$ are as stated there. Similarly, we may assume that $A^{\prime}=B^{\prime} \oplus D^{\prime}$. Since $\operatorname{det}(B)=f_{2}=\operatorname{det}\left(B^{\prime}\right)$, Lemma 4.4 implies that $B$ and $B^{\prime}$ are congruent. Since $D$ and $D^{\prime}$ have the same invariant factors, they are congruent by the induction hypothesis. Hence $A$ and $A^{\prime}$ are congruent.

\section{Canonical form under congruence}

In the next theorem we characterize the invariant factors of hermitian and skew-hermitian matrices. Clearly these factors have to be homogeneous.

Theorem 5.1. Let $0 \leq r \leq n$. Let $f_{1}, \ldots, f_{n}$ be a sequence of homogeneous elements in $R$ such that $f_{1}, \ldots, f_{r}$ are monic, each dividing the next one, and $f_{r+1}, \ldots, f_{n}$ are zero. Then this sequence is the list of invariant factors of a hermitian (resp. skew-hermitian) matrix $A \in M_{n}(R)$ of rank $r$ if and only if the following two conditions hold:

(i) Any maximal subsequence $f_{i}, f_{i+1}, \ldots, f_{j}$ consisting of consecutive nonzero odd (resp. even) elements has even length. We shall write such subsequence as

$$
g_{i}, h_{i}, g_{i+2}, h_{i+2}, \ldots, g_{j-1}, h_{j-1} .
$$

(ii) For each $\left(g_{k}, h_{k}\right)$ as above, $h_{k}=g_{k} p_{k} p_{k}^{*}$ with $p_{k}$ pure.

Proof. We prove necessity by induction on $n$. The cases $r=0$ and $n=1$ are trivial. Let $r \geq 1$ and $n \geq 2$. By replacing $A$ with $f_{1}^{-1} A$, we may assume that $f_{1}=1$.

If $A$ is hermitian, then Proposition 4.1 shows that $A$ is congruent to $(1) \oplus B$ and we can apply the induction hypothesis to $B$ to finish the proof.

If $A$ is skew-hermitian, then $A$ is congruent to the matrix $B \oplus D$ as stated in Proposition 4.2. In particular $f_{2}=\operatorname{det}(B)$ is even and not divisible by $t$. We can now finish the proof by applying the induction hypothesis to $D$.

Sufficiency can be read off from the next theorem.

It is now easy to obtain the canonical forms for hermitian and skew-hermitian matrices under congruence.

Theorem 5.2. Let $A \in M_{n}(R)$ and $A^{*}=\varepsilon A$, where $\varepsilon= \pm$, let $r$ be the rank of $A$, and let $f_{1}, \ldots, f_{n}$ be the invariant factors of $A$. Form the direct sum, $B$, of the following blocks: 
(i) The $1 \times 1$ matrices $\left(f_{i}\right)$ for each $f_{i}$ such that $f_{i}^{*}=\varepsilon f_{i}$.

(ii) The $2 \times 2$ matrices

$$
g_{k}\left(\begin{array}{cc}
0 & p_{k} \\
-p_{k}^{*} & 0
\end{array}\right)
$$

for each pair $\left(g_{k}, h_{k}=g_{k} p_{k} p_{k}^{*}\right)$ constructed in the previous theorem.

Then $A$ is congruent to $B$. Moreover such $B$ is unique up to the ordering of the diagonal blocks and the factorizations $h_{k}=f_{k} p_{k} p_{k}^{*}$.

Proof. The matrices $A$ and $B$ have the same invariant factors.

\section{Comments on other fields}

We introduce four conditions on a field $F$ assuming only that the characteristic is not 2 .

(K) Kac's question has affirmative answer for the field $F$.

(N) The norm map $R \rightarrow R_{0}$ sending $x \rightarrow x x^{*}$ is onto.

(U) The quadratic form $x^{2}-t y^{2}$ over $R$ is universal.

(I) No element of $R_{0}$ is irreducible in $R$.

Proposition 6.1. For a field $F$ of characteristic $\neq 2$, the above four conditions are equivalent to each other.

Proof. $(K) \Rightarrow(N)$. Let $\alpha \in F, \alpha \neq 0$. As

$$
A=\left(\begin{array}{cc}
-\alpha t & \alpha \\
-\alpha & t
\end{array}\right)
$$

is skew-hermitian but not diagonalizable, it must be congruent to

$$
\left(\begin{array}{cc}
0 & x \\
-x^{*} & 0
\end{array}\right)
$$

for some $x \in R$. Hence $\operatorname{det}(A)=-\alpha\left(t^{2}-\alpha\right)$ splits over $F$. We deduce that $F$ is quadratically closed, i.e., it has no quadratic extensions.

It remains to show that if $a=1+t^{2} b$, with $b \in R_{0}$, then $a=x x^{*}$ for some $x \in R$. This follows by applying the above argument to

$$
\left(\begin{array}{cc}
t b & 1 \\
-1 & t
\end{array}\right)
$$

$(N) \Rightarrow(K)$. Our proofs are valid under this weaker hypothesis.

$(N) \Rightarrow(U)$. Let $\sigma: R \rightarrow R_{0}$ be the isomorphism of $F$-algebras sending $t$ to $t^{2}$. For $b \in R$ we have $\sigma(b)=z z^{*}$ for some $z \in R$. By writing $z=\sigma(x)+t \sigma(y)$, $(x, y \in R)$, we obtain $\sigma(b)=\sigma(x)^{2}-t^{2} \sigma(y)^{2}$, i.e., $b=x^{2}-t y^{2}$.

$(U) \Rightarrow(N)$. For $a \in R_{0}$ we have $a=\sigma(b)$ with $b \in R$. As $b=x^{2}-t y^{2}$ for some $x, y \in R$, we have $a=z z^{*}$ with $z=\sigma(x)+t \sigma(y)$.

It is obvious that $(N)$ implies $(I)$ and the converse is not hard to prove. 
One can construct examples of fields $F$ satisfying the above conditions without being algebraically closed. Start with a finite Galois extension $K / E$ whose Galois group is not a 2-group. Let $\sigma$ be an $E$-automorphism of an algebraic closure $\bar{K}$ of $K$ whose restriction to $K$ is nontrivial and has odd order. Then one can take $F$ to be the quadratic closure of $(\bar{K})^{\sigma}$. In particular the quadratic closure of the prime field $F_{p}$ ( $p$ odd) is an example. On the other hand, it is easy to see that the quadratic closure of the rationals does not satisfy the condition (U).

In general, a hermitian or skew-hermitian matrix $A \in M_{n}(R)$ need not be congruent to the direct sum of any $1 \times 1$ or $2 \times 2$ matrices. For instance, this is the case when $F$ is the real field and

$$
A=\left(\begin{array}{ccc}
t^{2} & 1 & 0 \\
1 & t^{2} & t \\
0 & -t & t^{2}
\end{array}\right)
$$

\section{References}

[1] C. Boyallian, V. G. Kac, J. I. Liberati, On the classification of subalgebras of Cend ${ }_{N}$ and $\mathrm{gc}_{N}$, preprint: arXiv:math-ph/0203022

[2] D. Ž. Djoković, Hermitian matrices over polynomial rings, J. Algebra 43 (1976), 359-374.

[3] V.G. Kac, private communication, October 2001.

Department of Pure Mathematics, University of Waterloo, Waterloo, Ontario, N2L 3G1, CANADA.

E-mail address: djokovic@uwaterloo.ca

E-mail address: fszechtm@uwaterloo.ca 